\title{
Robust Spatial Data Hiding for Color Images*
}

\author{
Xiaoqiang Li, Xiangyang Xue, and Wei Li \\ Department of Computer Science and Engineering, \\ Fudan University, Shanghai 200433, China \\ xqli_fudan@hotmail.com
}

\begin{abstract}
In paper [1], we present a new watermarking technique in spatial domain expressly devised for color images, which is based on transmit diversity techniques. We also show that the robustness of our new algorithm is better than single color channel. However, we don't compare the robustness of our algorithm with luminance channel. In this paper, we describe the revised scheme of algorithm [1] in detail, and give experimental results that demonstrate the unmistakable advantage of our new approach over algorithms operating on not only single color channel, but also luminance channel. All experimental results prove that our scheme is more robust than any other single channel scheme in spatial for color images.
\end{abstract}

\section{Introduction}

The Internet and the World Wide Web have revolutionalized the way in which digital data is distributed. The wide spread and easy access to multimedia content has motivated development of technologies for data hiding, with emphasis on access control, authentication, and copyright protection. Much of the recent work in data hiding is about copyright protection of multimedia data. This is also referred to as digital watermarking. In the field of image watermarking, a great deal of research has been carried out for mainly two targets. The first is to enlarge the maximum number of information bits that can be hidden in a host image invisibly. The second is to improve the robustness of watermark. However, there is a trade-off between the capacity of the information embedded to a given image and the robustness of the watermarking system. Previous researches were mostly focused on grayscale image watermarking. For color images, researchers usually embed information in luminance channel [2,3], or in a single color channel [4,5]. Alternative approaches to color image watermarking have been advanced by Fleet and Heeger [6], who suggest embedding the watermark into the yellow-blue channel of the opponent-color representation of color images. The aim of their scheme is resisting printing and rescanning. Kutter et $a l$ in $[7,8]$ embed the watermark by modifying a selected set of pixel values in the blue channel, since human eye is less sensitive to changes in this band. However, the

\footnotetext{
*This work was supported in part by NSF of China under contract number 60003017, China 863 Projects under contract numbers 2001AA114120 and 2002AA103065.
} 
They didn't take this phenomenon into consideration. Barni et al [9] proposed a robust watermarking scheme for color images, its detection is based on a global correlation measure which is computed by taking into account the information conveyed by the three color channels as well as their interdependency, but it is only used to decide whether the watermark is exist or not. Multi-bits robust watermarking for image remains a topic of research. In [1], we developed a new multi-bits watermarking algorithm for color images based on transmit diversity techniques. In that scheme, color image is considered as three independent communication channels to meet the assumption of transmit diversity technique. We detect the transmitted information from combination of three channels. A single color channel may suffer severe fading in many cases. However, by using transmit diversity we can provide redundant replica of information to the extractor. Compared with the single channel systems, our scheme has better performance on capacity and on robustness to unintentional attacks, such as the JPEG lossy compression.

In this paper, we revise watermarking algorithm [1] in two aspects. Interleaver is replaced by shuffling in embedding algorithm. In extraction algorithm a simple method accumulating the energy of three channels is used. By this method, we can achieve the capacity much higher than that of scheme in [5]. Lots of experiments show that the watermarking scheme we present excel not only single color algorithm, but also luminance channel algorithm.

This paper is organized as follows. In the next section we give a representation of the transmit diversity technique. We combine this technique with watermarking. Embedding and extraction algorithm is described in section 3. In section 4, we prove by plentiful experiments that transmit diversity techniques in our scheme can make notable gains in both capacity and robustness. In section 5, we conclude that all these properties indicate that our scheme can be employed in the video watermarking and covert communication [11].

\section{Transmit Diversity and Watermarking}

Any single communication channel suffers fading aroused by various channel noises. As a result, the receiver must have a redundant replica of the transmitted signal for reliable communication. In wireless communications, transmit diversity techniques have been proposed for providing diversity gain for higher voice and data rates. By jointly designing the forward error correction coding, modulation, transmit diversity, and optional receiver diversity scheme, one can boost the throughput of band-limited wireless channels $[12,13]$.

In spatial spread spectrum watermarking scheme, a narrow-band signal (the watermark) has to be transmitted in a wide-band channel with noise (the image or video signal). Conventional algorithms just embed information into a single channel: one of the three color channels (always the blue one) or the luminance channel. According to the assumption in transmit diversity techniques, the RGB tones of color images should be considered as independent and slowly fading channels. Modulated by different pseudo-random sequences and shuffling respectively, the same watermarking in different forms is embedded into the three channels. We employ 
three receivers that can receive replicas of the same transmitted information through independent fading paths. Even if a particular path is severely faded, we are still able to recover a reliable estimate of the transmitted symbols through other propagation paths.

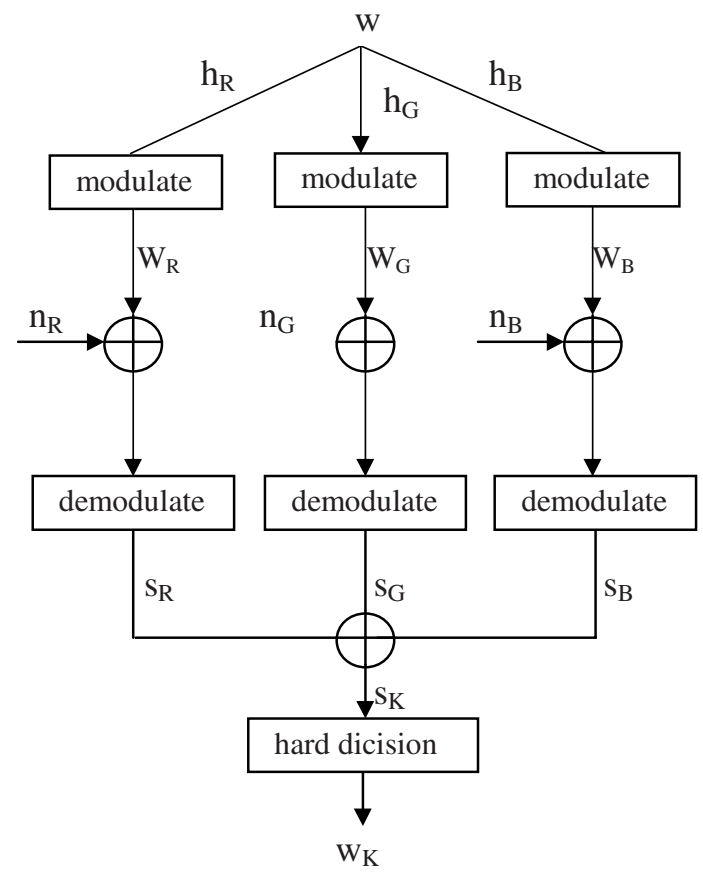

Fig. 1. Watermarking scheme based on the theory of transmit diversity techniques

Transmit diversity techniques fall into three main categories: information feedback-assisted schemes, feedforward or training assisted arrangements, and blind schemes [13]. As image is non-stationary pseudo signal, it is hard to find an appropriate channel model to describe its characteristics. We also find that the performance of the blue channel is not always better than the other two for all the color images. So we consider the images as unknown channel with unstructured interference. After demodulating the watermark from the three paths respectively, we decide the information bits by simply accumulating the energy of three channels. Figure 1 shows the spatial watermarking in conjunction with three propagation paths.

\section{Data Hiding in Spatial Domain}

Based on transmit diversity principle, new color image watermarking scheme embedding the same watermark in three color channels improves the decoding performance. To better hide the watermark to human observer, we use human visual system (HVS) model in spatial to ensure watermark invisible. 


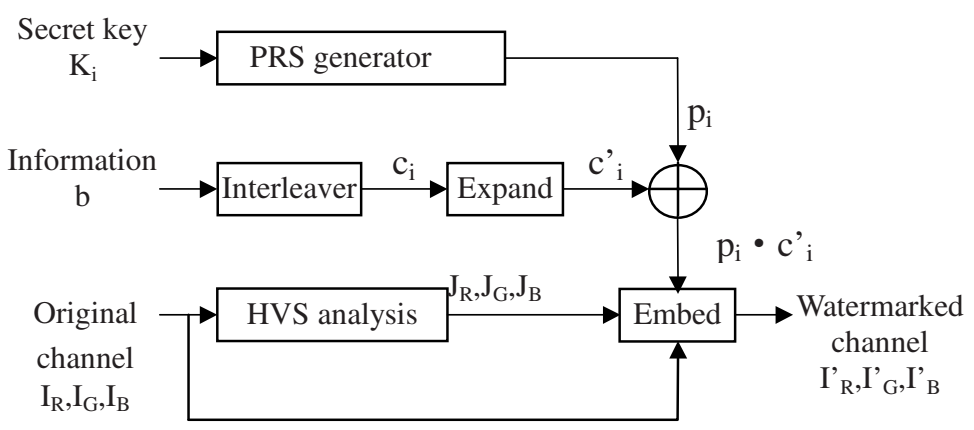

Fig. 2. Watermark embedding algorithm illustration

\subsection{Watermark Embedding Algorithm}

The embedding algorithm is shown in Figure 2. Given a color image $\boldsymbol{I}$, the $R, G, B$ channel are separated and denoted as $\boldsymbol{I}_{R}, \boldsymbol{I}_{G}, \boldsymbol{I}_{B}$. Hilbert scan is adopted and the twodimensional pixel sequence is reordered into a one-dimension sequence for each channel. Watermark $\boldsymbol{w}$ is represented by the binary antipodal vector $\boldsymbol{b}$. It is shuffled in different ways to be disordered into two new antipodal vectors which are denoted by $\boldsymbol{c}_{R}, \boldsymbol{c}_{G}$ respectively. Including the unshuffled information sequence itself, we get three antipodal vectors: $\boldsymbol{c}_{R}, \boldsymbol{c}_{G}, \boldsymbol{c}_{B}$. And these vectors should be expanded into three new vectors: $\boldsymbol{c}_{R}, \boldsymbol{c}_{G}, \boldsymbol{c}_{B}$. Then $\boldsymbol{c}_{R}{ }_{R}, \boldsymbol{c}_{G}, \boldsymbol{c}_{B}{ }_{B}$ are modulated into vectors: $\boldsymbol{p}_{R} \cdot \boldsymbol{c}_{R}, \boldsymbol{p}_{G} \cdot \boldsymbol{c}_{G}$, $\boldsymbol{p}_{B} \cdot \boldsymbol{c}^{\prime}{ }_{B}$ by three different pseudo-random antipodal sequences $\boldsymbol{p}_{R}, \boldsymbol{p}_{G}, \boldsymbol{p}_{B}$ produced by pseudo-random-sequence (PRS) generator, which are controlled by three secrete keys: $\mathrm{K}_{\mathrm{R}}, \mathrm{K}_{\mathrm{G}}, \mathrm{K}_{\mathrm{B}}$ for security purpose. Considering the invisibility character, we use a human visual system (HVS) analyzer to control the watermark strength. It calculates the Just Noticeable Distortion (JND) mask: $\boldsymbol{J}_{\boldsymbol{R}}, \boldsymbol{J}_{G}, \boldsymbol{J}_{B}$, for each color channel of the host image respectively. Multiplied by the JND, each modulated vector is embedded into corresponding color channel according to the following rule:

$$
\boldsymbol{I}_{i}^{\prime}=\boldsymbol{I}_{i}+\boldsymbol{J}_{i} \cdot \boldsymbol{p}_{i} \cdot \boldsymbol{c}^{\prime}{ }_{i}
$$

where $i \in\{\mathrm{R}, \mathrm{G}, \mathrm{B}\}$. The watermarked color image $I$ ' is obtained by reassembling $\boldsymbol{I}_{R}$, $I_{G}, I_{B}{ }^{\prime}$

To maximize the admissible amplitude of the unperceivable information embedded, we use the HVS model in [5] and calculate the JND mask of each color channel directly in the spatial domain. This algorithm contains 3 aspects: texture and edge analysis, edge separation and reclassification, luminance sensitivity analysis. Firstly, each channel of the original image is divided in blocks of $8 \times 8$ pixels. Then to compute JND matrix for each $8 \times 8$ pixels block as follow:

$$
J_{i}(x, y)=l_{i}(x, y)+\operatorname{dif}_{i}(x, y)
$$

where $i \in\{\mathrm{R}, \mathrm{G}, \mathrm{B}\}$. For each channel, $l_{i}(x, y)$ represents the additional noise threshold and $\operatorname{dif}_{i}(x, y)$ represents the basic noise threshold of the block it belongs to. Each JND mask has the same size with the original image, denoted as $\boldsymbol{J}_{\boldsymbol{R}}, \boldsymbol{J}_{\boldsymbol{G}}, \boldsymbol{J}_{\boldsymbol{B}}$. 


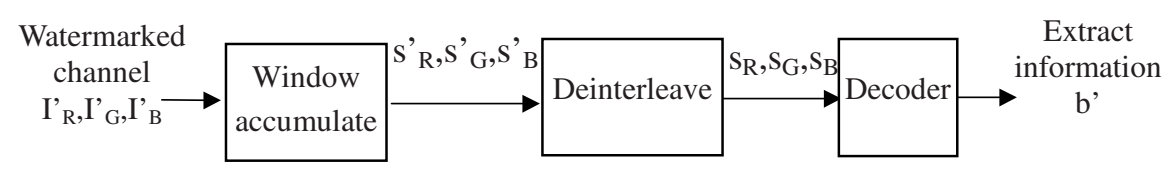

Fig. 3. Watermark extraction algorithm illustration

\subsection{Shuffling Algorithm}

There are three advantages we use shuffling in our scheme. First, the watermark is shuffled in different ways can better meet the assumption of transmit diversity technique than original scheme [1]. Second, after shuffling, information bit is scattered in different area of different color channel. So the same information bit is possibly embedded in flat region and texture region, and we can make use of texture region to recover more bit correctly in extract process. Third, shuffling also enhances security since the shuffling table or a key for generating the table is needed to correctly extract the hidden data.

A key $k=\left(k_{0}, k_{1}\right)$ is chosen by the copyright owner, where $k_{0}$ is an arbitrary integer, and $k_{1}$ is an integer within the interval [N/3, 2N/3] and is prime to $N$. Define

$$
f(i)=\left(k_{0}+k_{1}\right) \bmod N, i=0,1, \ldots, \mathrm{N}
$$

Clearly, a one-to-one mapping between $i$ and $f(i)$ exists. In extraction procedure, we can derive $i$ from $f(i)$ by using above algorithm on $f(i)$. Define

$$
i=\left(f(i)-k_{0}\right) \times k_{2} \bmod N
$$

where $k_{2}$ satisfies the equation (4)

$$
k_{2} \times k_{1}=1 \bmod N
$$

\subsection{Watermark Extraction Algorithm}

The extraction algorithm is shown in Figure 3. For a given watermarked color image $\boldsymbol{I}$, the channel $\boldsymbol{I}_{R}, \boldsymbol{I}_{G}, \boldsymbol{I}_{B}{ }_{B}$ are first separated. Hilbert scan is used again to transfer two-dimensional sequences into one-dimensional sequences. Then we calculate the window energy $\mathrm{s}_{i, k}[1]$ for each channel. Before deciding the extracted information bit, we synchronize the three channels by deshuffling $s_{R}, s_{G}, s_{B}{ }_{B}$ into $s_{R}, s_{G}, s_{B}$. For computational simplicity, we use simple following methods to decide the information bits:

Meth--accumulating the value of $s_{R, k}, s_{G, k}, s_{B, k}$ for each bit, the sum is denoted by $s_{k}$.

After getting $s_{k}$, we use hard-decision to estimate the message according the sign of $s_{k}$, namely:

- If the sign of $s_{k}$ is positive, the extracted information bit is 1 ;

- If the sign of $s_{k}$ is negative, the extracted information bit is 0 . 


\section{Experimental Results}

Here we just give some experiment results for brevity. Please note that watermarks in five schemes are the same in all aspects except the channels in which they are embedded. We have tested the proposed algorithm on images with various content complexity, e.g., "Lena," "Pepper," "Baboon," and "F16." All these images are $512 \times 512 \times 24$ color image. The image editing and attacking tools adopted in experiment are StirMark 4.0 and Photoshop 6.0.

\subsection{Capacity Analysis}

Watermarking based on direct-sequence spread spectrum in spatial domain is a highpayload technique. But we have to trade off bandwidth and capacity in reality. As transmit diversity can improve the throughput of band-limited wireless channel, it may operates on watermark-channel. Experiments show that a certain color channel doesn't perform congruously. So referring to the transmit diversity principle, we model the three color tones of RGB image as three independent fading channels and embed the watermark in three channels simultaneously. Extensive experiments prove that this method can furthest integrate the merit of each channel. When embedding the same capacity of information, the BER of new algorithm is always lower than that of single channel scheme (See Figure 4) for all the four images.

In Huan's [10] experiment, 192 information bits are embedded and watermark extraction need original unwatermarked image. Lots of experiment result proves that propose blind watermarking algorithm in the paper is able to hide 512 bits without error. Du [5] mainly studied four kinds of convolutional code with different constraint lengths by comparing their performance on BER with that of the uncoded scheme in single channel. Combined convulutional code with proposed algorithm, the capacity can be improved again.

\subsection{Robustness Test}

To test the robustness against noise attack, we distorted the watermarked "Peppers" by Gaussian noise with different amount, and the peak signal-to-noise (PSNR) correspond to different amount noise is listed in Table 1. If the noise amount is $2 \%$, the BER when using red, green, blue and luminance channel separately is $2.53 \%, 4.88 \%$ and $1.17 \%$. From Figure 5, we can see that the BER of our algorithm is only $2 \%$ when the noise amount is equal $20 \%$, but it's more than $7 \%$ for any single channel. Especially when the noise amount is below $10 \%$, our scheme can keep the BER almost zero. Experiment results prove that our algorithm is considerably more robust than single channel. 
Table 1. The amount of Gaussian noise (in percentage) vs. PSNR in each channel

\begin{tabular}{cccccccccccc}
\hline $\begin{array}{c}\text { PSNR } \\
(\mathrm{db})\end{array}$ & \multicolumn{8}{c}{ The amount of Gaussian noise (\%) for watermarked image "Peppers" } \\
\cline { 2 - 12 } & 2 & 4 & 6 & 8 & 10 & 12 & 14 & 16 & 18 & 20 \\
\hline $\mathrm{R}$ & 38.73 & 32.77 & 29.13 & 26.87 & 24.52 & 22.76 & 21.78 & 20.88 & 19.97 & 19.05 \\
\hline $\mathrm{G}$ & 38.69 & 32.74 & 29.16 & 26.96 & 24.66 & 22.94 & 22.06 & 21.13 & 20.25 & 19.30 \\
\hline $\mathrm{B}$ & 38.68 & 32.79 & 29.21 & 27.03 & 24.72 & 22.98 & 22.07 & 21.16 & 20.15 & 19.23 \\
\hline
\end{tabular}

Using transmit diversity techniques, we greatly improve the robustness to the unintentional distortion as JPEG compression. In our experiments, we fix the hiding rate at 1 bit per 1024 pixels. It can be seen in Figure 6 that our scheme is more robust to JPEG compression than single channel schemes.

We test the performance on resistance to cropping by cutting off the borders of the watermarked images and experiment results in Table 2 shows that new scheme performs much better than the single channel schemes. When the watermark is transmitted through a single channel, information bits in the regions that are cut off can't be extracted correctly. But we can recover these bits by replica transmitted in other channels. In addition, due to shuffling, the information bits in a certain channel of the cropped region are undestroyed in other channels because they are embedded in other regions that in most cases are not cropped.

Our scheme can also resist low-pass filtering at certain degree. We have tested the robustness against median filter with $3 \times 3$ window size. We find that our scheme shows great advantage on the robustness to low-pass filtering when only one color channel is filtered. Experiment results are shown in Table 3, where $\mathrm{B}_{\mathrm{f}}$ means to filter the blue channel.

Table 2. BER (\%) of five schemes for cropped watermarked image "Peppers" with 512 information bits

\begin{tabular}{cccccc}
\hline \multirow{2}{*}{$\begin{array}{c}\text { Embedded } \\
\text { Channel }\end{array}$} & \multicolumn{5}{c}{$\begin{array}{c}\text { Cut off borders to image "Peppers" } \\
\text { (\% of image cropped) }\end{array}$} \\
\cline { 2 - 6 } & $5 \%$ & $10 \%$ & $15 \%$ & $20 \%$ & $25 \%$ \\
\hline $\mathrm{R}$ & 5.85 & 9.76 & 13.47 & 13.86 & 21.87 \\
\hline $\mathrm{G}$ & 6.05 & 9.17 & 13.67 & 13.47 & 24.20 \\
\hline $\mathrm{B}$ & 2.14 & 5.27 & 9.17 & 9.17 & 16.70 \\
\hline Lum & 5.85 & 8.65 & 11.58 & 14.04 & 16.79 \\
\hline Meth & 0 & 0.19 & 0.39 & 0.58 & 0.97 \\
\hline
\end{tabular}

\section{Conclusion}

In this paper, we describe the revised scheme of watermarking algorithm [1] in detail, and give experimental results that demonstrate the unmistakable advantage of our new approach over algorithms operating on not only single color channel, but also 
luminance channel. Experimental result also prove that our scheme is more robust than any other single channel scheme in spatial for color images. This algorithm cost little time on extraction so that it can be applied to video watermark. Its robustness to kinds of attacks also makes it suitable for broadcast monitoring and covert communication.

Proposed algorithm is blind data hiding scheme, information bits can be extracted without original image, but it is fragile to geometric-based attack. So our further work focuses on designing a method to successfully resist geometric-based attack.

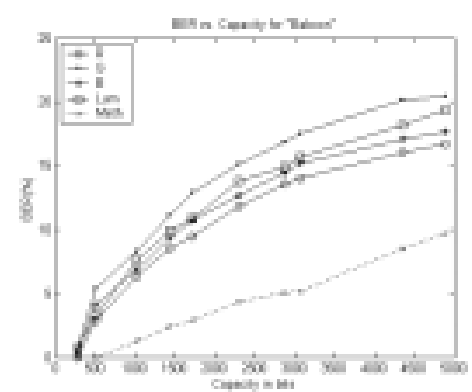

(a)

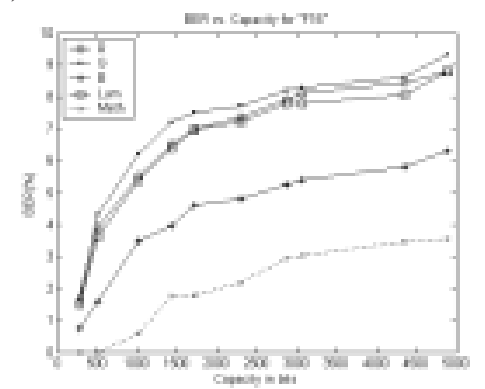

(c)

Fig. 4. Capacity test for image "Baboon", "F16", "Lena" and "Peppers". Detection BER (\%) for watermarked image with different amount of information bits.

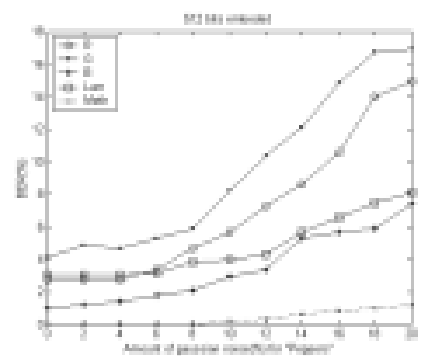

Fig. 5. Empirical BER with respect to the noise (in percentage) with PSNR listed in Table 1 for "Pepper"

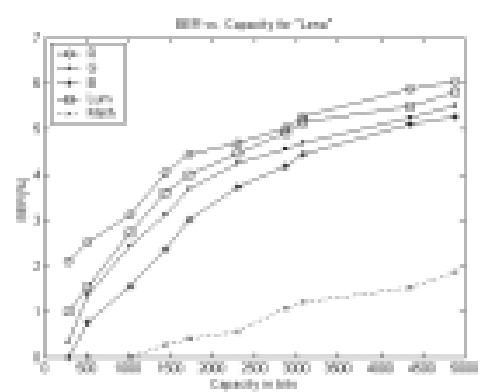

(b)

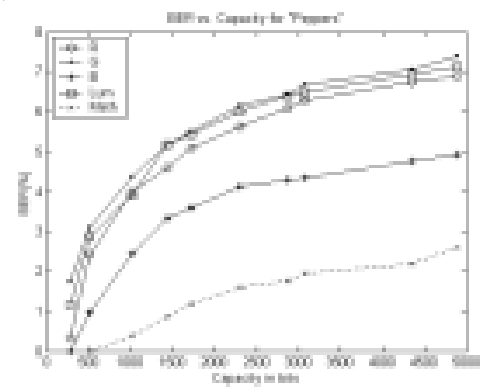

(d)

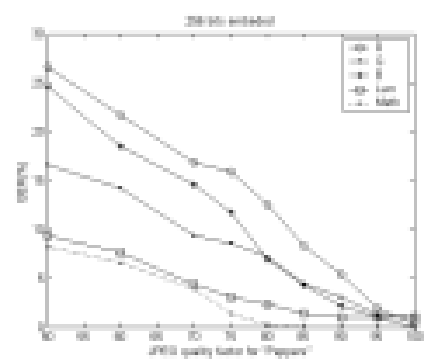

Fig. 6. Empirical BER with respect to the JPEG compression with different quality factor for "Pepper" 
Table 3. BER $(\%)$ of five schemes after $3 \times 3$ median filtering for "Peppers"

\begin{tabular}{cccccc}
\hline $\begin{array}{c}\text { Watermark } \\
\text { (bit) }\end{array}$ & Meth & Lum & $\mathrm{R}$ & $\mathrm{G}$ & $\mathrm{B}$ \\
\cline { 2 - 6 } & $\mathrm{B}_{\mathrm{f}}$ & $\mathrm{B}_{\mathrm{f}}$ & $\mathrm{R}_{\mathrm{f}}$ & $\mathrm{G}_{\mathrm{f}}$ & $\mathrm{B}_{\mathrm{f}}$ \\
\hline 256 & 0 & 0.21 & 5.85 & 3.15 & 3.52 \\
\hline 512 & 0.39 & 3.13 & 23.04 & 22.41 & 16.99 \\
\hline 1024 & 0.66 & 4.63 & 22.55 & 22.41 & 22.24 \\
\hline
\end{tabular}

\section{References}

1. X.Q. Li, Z.Y. Du, et al. "Multi-channel Data Hiding Scheme for Color Images", in Proceeding of International Conference on Information Technology: Coding and Computing, ITCC2003, Apr 28, 2003.

2. W. Bender, D. Gruhl, and et al, "Technique for Data Hiding", IBM Systems Journal, vol. 35, no.3\&4, pp.313-336, 1996

3. G. C. Langelaar, J. C. A. Van der Lubbe, and R. L. Lagendijk, "Robust Labeling Methods for Copy Protection of Images", in Storage and Retrieval for Image and Video Databases V, I. K. Sethin and R. C. Jain, Eds. San Jose, CA: IST and SPIE, Feb. 1997, vol. 3022, pp.298-309

4. N. Nikolaidis and I. Pitas, "Robust Image Watermarking in the Spatial Domain", Signal Processing, vol. 66, no. 3, pp. 385-403, May 1998.

5. Z.Y. Du, Y. Zhou, and P.Z. Lu, "An Optimized Spatial Data Hiding Scheme Combined with Convolutional Codes and Hilbert Scan”, PCM2002.

6. D. Fleet and D.Heeger, "Embedding Invisible Information in Color Images", in Proc. IEEE Inter. Conf. Image processing'97, Santa Barbara, CA, October 26-29 1997, vol.1, pp.532-535.

7. M. Kutter, F. Jordan, and F. Bossen, "Digital signature of Color Images Using Amplitude Modulation" in Storage and Retrieval for Image and Video Database V, SPIE vol. 3022, San Jose, CA, February 8-14 1997, pp. 518-526

8. M. Kutter, S. Winkler, "A Vision-based Masking Model for Spread-spectrum Image Watermarking", IEEE Tran. on Image Processing, vol.11, no.1, Jan. 2002

9. M. Barni, F. Bartolini and A. Piva, "Multichannel watermarking of color images", IEEE Transactions on circuits and systems for video technology, vol. 12, no. 3, pp.142-156, Mar. 2002.

10. J. Huang and Y.Q. Shi, "Reliable Information bit hiding", IEEE Transactions on circuits and systems for video technology, vol. 12, no. 10, pp. 916-920, Oct. 2002.

11. Ingemar J.Cox, Matt L. Miller and Jeffrey A. Bloom, "Watermarking Applications and Their Properties", in Proceeding of Int. Conf. On Information Technology'2000, Las Vegas, 2000.

12. V. Tarokh, N. Seshadri and A.R. Calderbank, "Space-time codes for high data rate wireless communication: performance criterion and code construction", IEEE Transactions on Information Therory, vol. 44, no. 2, Mar. 1998.

13. T. H. Liew and L. Hanzo, "Space-time Black Codes and Concatenated Channel Codes for Wireless Communications", in Proceedings of the IEEE, vol.96, no.2, pp. 744-765, Feb. 2002 . 